\title{
Corticospinal Sprouting Differs According to Spinal Injury Location and Cortical Origin in Macaque Monkeys
}

\author{
(D) Corinna Darian-Smith, Alayna Lilak, Joseph Garner, and Karen-Amanda Irvine \\ Department of Comparative Medicine, Stanford University School of Medicine, Stanford, California 94305-5342
}

\begin{abstract}
The primate corticospinal tract (CST), the major descending pathway mediating voluntary hand movements, comprises nine or more functional subdivisions. The role of subcomponents other than that from primary motor cortex, however, is not well understood. We have previously shown that following a cervical dorsal rhizotomy (Darian-Smith et al., 2013), CST projections originating from primary somatosensory (S1) and motor (M1) cortex responded quite differently to injury. Terminal projections from the S1 (areas 3b/1/2) shrank to $<60 \%$ of the contralateral side, while M1 CST projections remained robust or expanded ( $>110 \%$ ). Here, we asked what happens when a central lesion is added to the equation, to better simulate clinical injury. Monkeys $(n=6)$ received either a unilateral (1) dorsal root lesion (DRL), (2) or a combined DRL/dorsal column lesion (DRL/DCL), or (3) a DRL/DCL where the DCL was made 4 months following the initial DRL. Electrophysiological recordings were made in S1 4 months postlesion in the first two groups, and 6 weeks after the DCL in the third lesion group, to identify the reorganized region of D1-D3 (thumb, index finger, and middle finger) representation. Anterograde tracers were then injected bilaterally to assess spinal terminal labeling. Remarkably, in all DRL/DCL animals, terminal projections from the S1 and M1 extended bilaterally and caudally well beyond terminal territories in normal animals or following a DRL. These data were highly significant. Extensive sprouting from the S1 CST has not been reported previously, and these data raise important questions about S1 CST involvement in recovery following spinal injury.
\end{abstract}

Key words: axonal sprouting; corticospinal pathway; motor cortex; nonhuman primate; somatosensory cortex; spinal cord injury

\section{Introduction}

The corticospinal tract (CST) in the macaque monkey and higher primates is the primary pathway involved in volitional movements of the hands (for review, see Schieber, 2007; Lemon, 2008; Isa et al., 2013), and its importance peaks in humans where finger control is most sophisticated. In macaques, the pathway innervating the cervical cord has $\geq 9$ different cortical origins (Catsman-Berrevoets and Kuypers, 1976; Murray and Coulter, 1981; Nudo and Masterton, 1990; Dum and Strick, 1991; Galea and Darian-Smith, 1994; Darian-Smith et al., 1996; Lemon, 2008), including primary motor (M1), primary somatosensory (S1), premotor, and other cortices. This number may be greater in humans. The spinal termination pattern for each cortical region is unique, reflecting a different functional role in hand use (Ralston and Ralston, 1985; Lemon and Griffiths, 2005; Morecraft et al., 2013).

The M1 CST is known to play an important role in the recovery of hand function following hemisection lesions in monkeys

\footnotetext{
Received April 14, 2014; revised July 14, 2014; accepted July 29, 2014

Author contributions: C.D.-S. designed research; C.D.-S., A.L., and K.-A.I. performed research; C.D.-S., A.L., J.G., and K.-A.I. analyzed data; C.D.-S. wrote the paper.

This work was supported by the National Institutes of Health National Institute of Neurological Disorders and Stroke (R01 NS048425). We also thank Jerome Geronimo for his technical assistance.

The authors declare no competing financial interests.

Correspondence should be addressed to Corinna Darian-Smith, Department of Comparative Medicine, 300 Pasteur Drive, Edwards Building Room R350, Stanford University School of Medicine, Stanford, CA 94305-5342. E-mail: cdarian@stanford.edu.

DOI:10.1523/JNEUROSCI.1593-14.2014

Copyright $\odot 2014$ the authors $\quad 0270-6474 / 14 / 3312267-13 \$ 15.00 / 0$
}

(Galea and Darian-Smith, 1997a,b; Rosenzweig et al., 2010). Rodent studies likewise indicate CST regenerative potential following experimental manipulations (Brus-Ramer et al., 2007; García-Alías et al., 2009; Ghosh et al., 2010; Liu et al., 2010), though the contribution of CST sensorimotor subcomponents is not known, and other tracts (i.e., rubrospinal) may be equally important in recovery.

We have previously shown in macaques, following a unilateral cervical dorsal root lesion (DRL) that removes input from the first three digits (D1-D3), (1) that there is an initial impairment, but subsequent recovery, of digit use over several months (Darian-Smith and Ciferri, 2005); (2) that behavioral recovery is accompanied by local sprouting of spared primary afferents within the dorsal horn (Darian-Smith, 2004); (3) that there is considerable reorganization of the "digit" maps within the cuneate nucleus and S1 (Darian-Smith and Brown, 2000; DarianSmith, 2009); and (4) that there are major spinal synaptic changes (Darian-Smith et al., 2010). We have also shown that M1 and S1 subcomponents of the CST respond quite differently following a DRL (Darian-Smith et al., 2013), whereby the M1 CST sprouts into the dorsal horn on the side of the lesion, and the S1 CST shrinks to $<60 \%$ of its original size. This suggests that, following a DRL, the M1 CST plays an important role in postinjury recovery, while the involvement of the S1 CST declines.

Here we were interested in the specific role of S1 and M1 CST projections in postinjury recovery when a central injury is added to the equation, since this model better simulates clinical injuries (e.g., dorsal root avulsions or any spinal injury involving a pe- 
Table 1. Details of monkeys used in the study

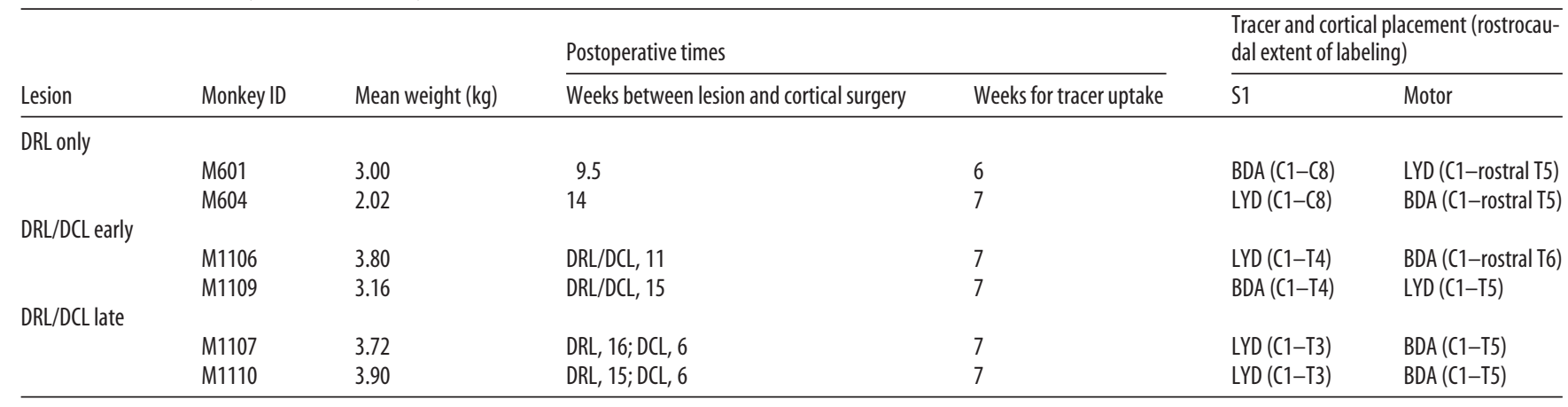

DRL/DCL early animals received both lesions at the same time, whereas DRL/DCL late monkeys received the DCL $\sim 4$ months following the initial DRL

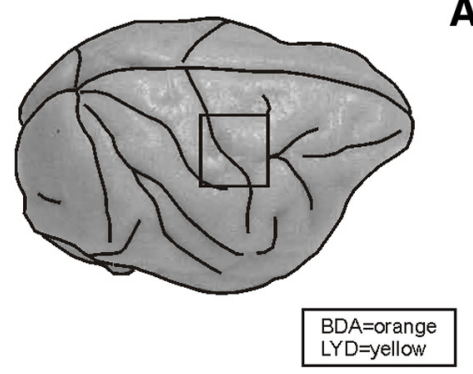

Left side
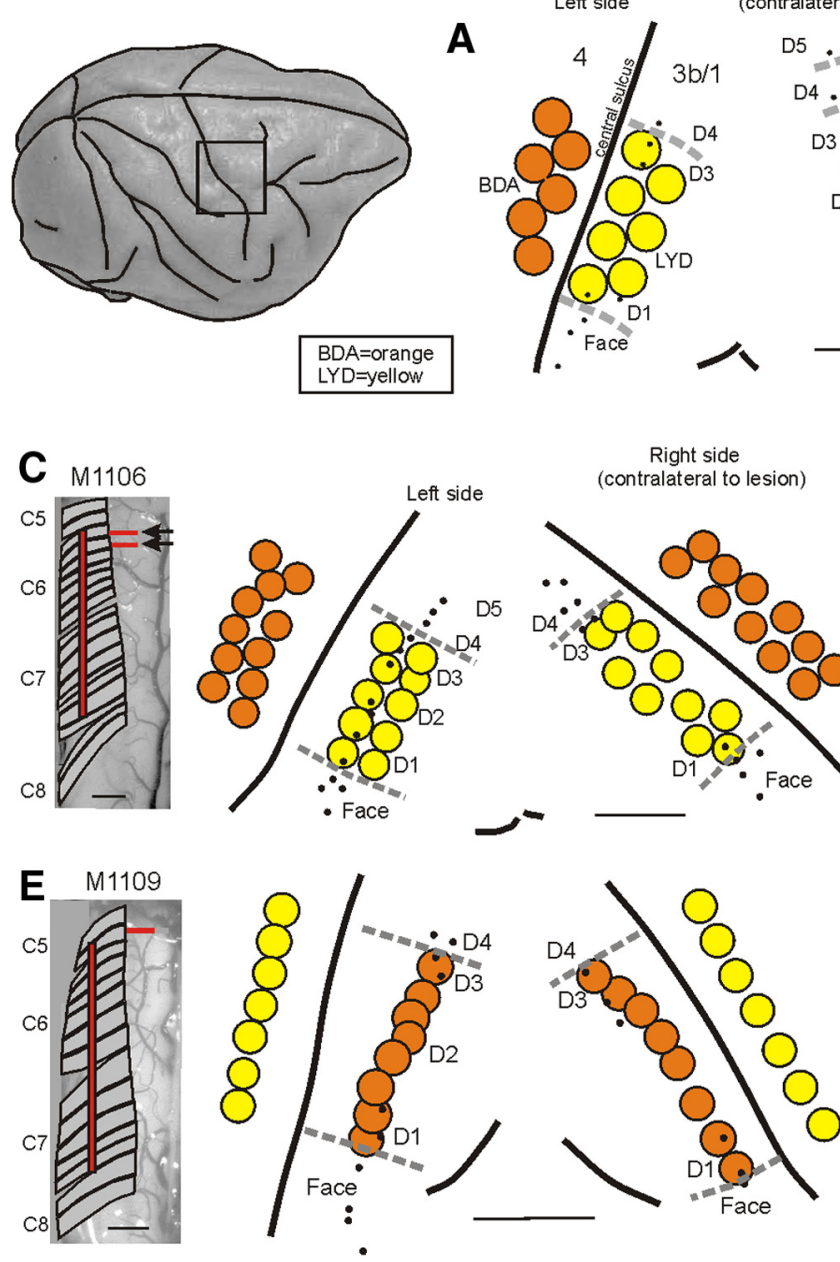

Right side (contralateral to lesion)
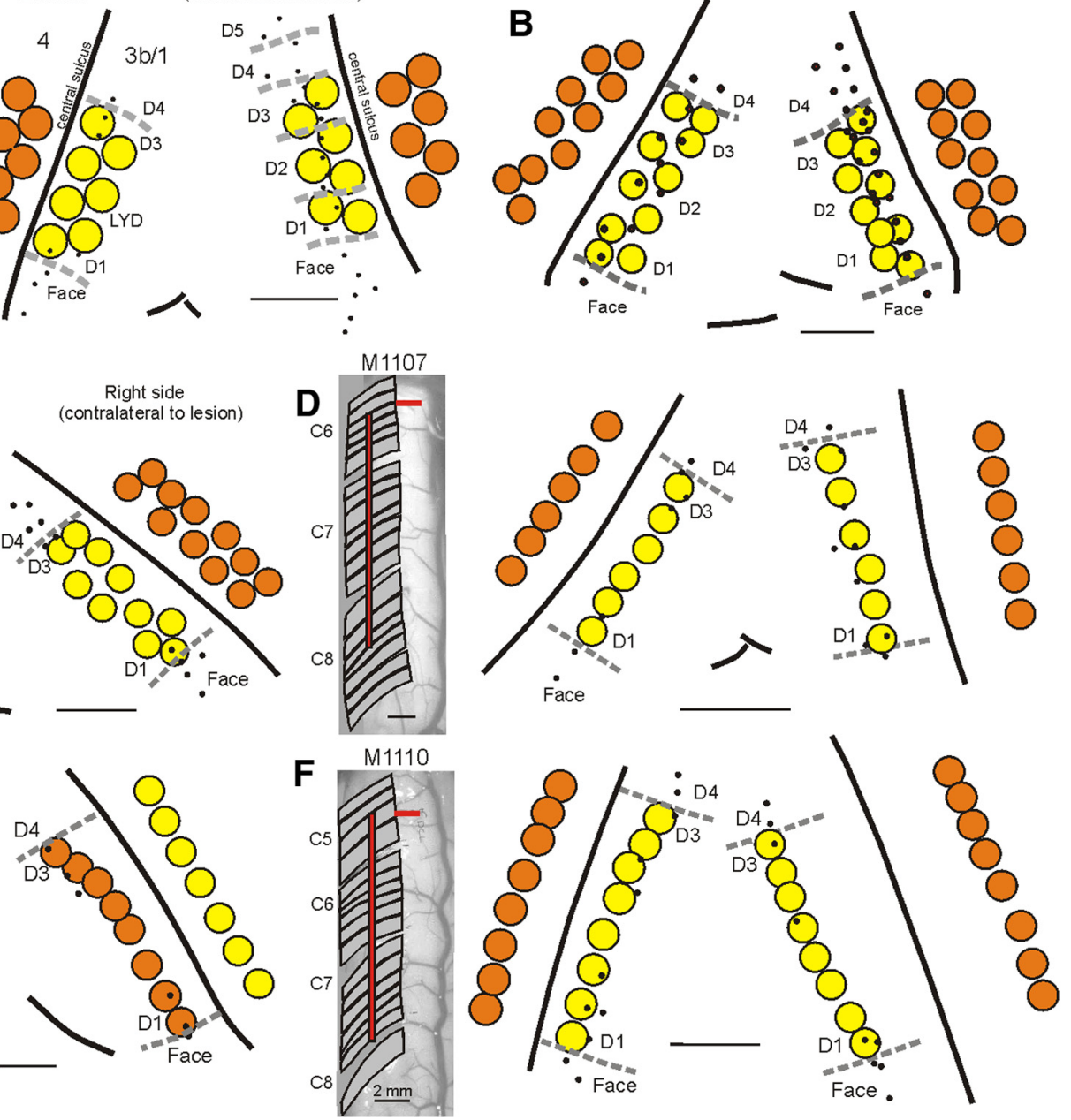

Figure 1. Placement of spinal cord DRL and DCL lesions (left side in all monkeys), and cortical maps showing recording sites and neuronal tracer placement. Motor cortex injections were placed according to somatosensory cortex recordings. Sections were processed through all injections to ensure that white matter was not involved and that there was no cross-sulcal contamination. No visible damage or necrosis was observed at the injection sites. Individual injection site volumes were not estimated, given poor definition of the injection borders of the tracers LYD (yellow) and BDA (red). However, injections were found to be equivalent in size and placement across animals. Scale bars, $2 \mathrm{~mm}$.

ripheral and central component). We were also interested in the effect of a small central injury made months following a DRL, since a delay in its timing provides mechanistic insight into the chronic condition and suggests therapeutic strategies that may help optimize postinjury recovery. Our findings show dramatic changes following a combined DRL/dorsal column lesion (DCL), regardless of whether the DCL was made at the same time as the DRL or 4 months later. In both scenarios, the S1 and M1 CST both sprouted dramatically to (potentially) form new connections. In this way each is likely to be contributing to circuitry remodeling, and each plays a key role in the recovery process.

\section{Materials and Methods}

Animals used and general procedures. Six young adult male macaque monkeys (Macaca fascicularis), averaging $3.3 \mathrm{~kg}$ (Table 1), were used in this study. All monkeys were colony bred and between 3 and 4 years of 
DRL/DCLearly

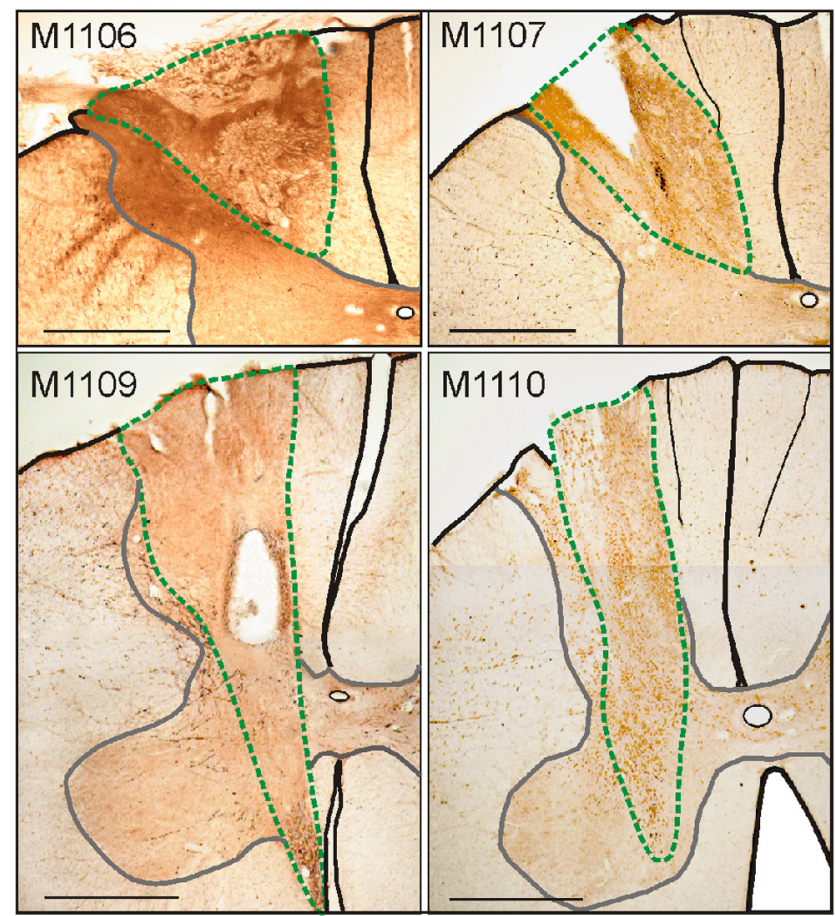

Figure 2. Photomicrographs showing the core of the $D C L$ made in each of the DRL/DCL monkeys in the present study. Sections were taken from the series stained for Lucifer yellow with DAB. Note that in all cases, the lesion was confined to the cuneate fasciculus, so afferent inputs from the trunk and hindlimb were left intact. M1106 and M1109 were early lesions. M1107 and M1110 were late lesions. Scale bars, $1 \mathrm{~mm}$.

age (where sexual maturity is typically reached at $4-5$ years in this species). Though the animals used in the study were relatively young, their sensorimotor pathways mediating hand function were considered mature, and their age is unlikely to have played a significant role in our findings. Monkeys were housed individually at the Stanford Research Animal Facility, in four-unit cages for each monkey $(64 \times 60 \times 77 \mathrm{~cm}$ depth $\times$ width $\times$ height per unit). All procedures were conducted in accordance with National Institutes of Health guidelines and approved by the Stanford University Institutional Animal Care and Use Committee.

Experimental sequence. Monkeys were divided into three groups. Two animals received a DRL alone, and data from these monkeys were reported previously (Darian-Smith et al., 2013). Four monkeys (not used elsewhere) received combined DRL/DCLs but were divided into two groups based on whether the central DCL was made at the same time as the DRL $(n=2)$ or 4 months following an initial DRL $(n=2)$. This delay provided additional insight into the role of the central injury in the CST response. To make lesions (Figs. 1,2), a laminectomy was used to expose the cervical cord to assess the distribution of sensory input from the first three digits of one hand (the thumb, index finger, and middle fingers or D1-D3). Dorsal rootlets innervating D1-D3 were mapped electrophysiologically and cut to produce the DRL. To make the DCL, the cuneate fasciculus was targeted and cut (Fig. 2). In the final surgery in all monkeys (for timing details, see Table 1), a bilateral craniotomy was made over the $\mathrm{S} 1$ and M1 in the region of hand representation. This area was mapped to locate the boundaries of D1-D3, and a series of injections of either the anterograde tracer biotin dextran amine (BDA; Sigma-Aldrich, B-9139), or Lucifer yellow dextran (LYD; Invitrogen, A5750) were made into the S1 or M1 cortex (Fig. 1). Figure 1 and Table 1 specify which tracer was used in each cortical region in each monkey.

Surgery and perfusion. Anesthesia was first induced with ketamine hydrochloride $(10 \mathrm{mg} / \mathrm{kg})$ and animals were subsequently maintained throughout surgery with gaseous isoflurane $(1-2 \%) / \mathrm{O}_{2}(1 \%)$ using a standard open circuit anesthetic machine. Atropine sulfate $(0.05 \mathrm{mg} / \mathrm{kg}$, i.m.) and cefazolin (20 mg/kg, i.v.) were administered before surgery. A laminectomy was made during the first surgery in each monkey and the overlying dura cut to expose dorsal rootlets from segments C5-T1. Electrophysiological recordings were made to create a microdermatome map (Darian-Smith and Brown, 2000; Darian-Smith, 2004; Darian-Smith and Ciferri, 2005; Darian-Smith et al., 2013), and this was then used to select rootlets to cut. All rootlets with receptive fields (RFs) on D1-D3 were cut in two places (using iridectomy scissors) to remove $2-3 \mathrm{~mm}$ of the rootlet along the length of the lesion. A DCL was made level with the most rostral cut rootlet (Fig. 1), using a scalpel blade (no. 11) that had been scored at $2 \mathrm{~mm}$ from the tip. The overlying tissues and skin were then closed in layers. Buprenorphine $(0.01-0.02 \mathrm{mg} / \mathrm{kg}, \mathrm{i} . \mathrm{m}$.) was administered after suturing to provide a postoperative analgesic, and monkeys were returned to their cages and observed closely. Monkeys were awake and alert typically within $1 \mathrm{~h}$, and no postoperative sequelae were observed.

In the cortical surgery, a bilateral craniotomy was made over the central sulcus and electrophysiological recordings (in S1) were used to identify the boundaries of the D1-D3 representation for tracer injection (contralaterally, this was the region of cortical reorganization). A small $\sim 1 \mathrm{~cm}^{2}$ bone flap was removed and the dura opened as a series of small windows. This helped maintain a healthy cortex over several hours. Agar (3\% in physiologic saline) was used to dampen cortical movement for recording but then removed before tracer injection. Once tracer injections were completed, the bone flap was replaced and the overlying skin sutured. All anesthesia and postoperative analgesic regimens were as described for the laminectomy. Monkeys also recovered rapidly from this procedure.

At the end of each experiment, monkeys were deeply anesthetized and given a lethal dose (intravenously) of sodium pentobarbital $(0.44 \mathrm{ml} / \mathrm{kg})$ until spontaneous breathing and corneal reflex had ceased. Animals were then manually breathed and perfused transcardially with heparinized $0.1 \mathrm{M}$ PBS, pH 7.4, followed by 4\% paraformaldehyde in phosphate buffer (PB), $\mathrm{pH}$ 7.4. The brain and spinal cord were removed, postfixed (same perfusate) for $4 \mathrm{~h}$, and transferred to $30 \%$ sucrose for cryoprotection.

Electrophysiological recordings in dorsal roots and cortex. Recordings were made from dorsal rootlets (Darian-Smith and Brown, 2000; Darian-Smith, 2004; Darian-Smith and Ciferri, 2005). Briefly, a laminectomy was made and dura resected to expose rootlets from C5-T1. The exposure was photographed so that individual rootlets could be identified for accuracy. A tungsten microelectrode $(1.2-1.4 \mathrm{~m} \Omega$ at $1 \mathrm{kHz})$ was used to record from each rootlet, and 8-10 single or small multiunit extracellular recordings were made from axons within each fascicle to produce a microdermatome map. Cutaneous RFs were mapped by progressively using hand manipulation, a camel hair brush, and Von Frey hairs. The RF was defined as cutaneous if the stimulus force of $\leq 2.0 \mathrm{~g}$ evoked a response. When the stimulus force of the Von Frey hair was $>2.0 \mathrm{~g}$, or where joint movement or hand manipulation were needed to evoke a response, the RF was marked as deep. Where there was ambiguity as to whether the RF was cutaneous or deep, the RF was included as cutaneous for the purposes of defining which rootlets to lesion. All RFs were mapped on to hand/body image score sheets and rootlets with cutaneous RFs on the thumb, index finger, and middle finger were then cut.

Cutaneous RF maps of hand representation were also made bilaterally within the S1 (6-7 weeks before the end of the experiment; Table 1). S1 in this study refers to Brodmann areas $3 \mathrm{~b}$ and 1 , and possibly the rostral tip of area 2 in some monkeys. In M1109, illustrated in Figure 3, the injection sites were contained within cytoarchitectonic regions $3 \mathrm{~b}$ and 1 . Area $3 \mathrm{a}$ was not involved in any of our analyses. A craniotomy was made and dura cut to expose a small window of cortex $\left(\sim 1 \mathrm{~cm}^{2}\right)$. Electrode penetrations were then made along the rostral lip of the postcentral gyrus at the approximate border of Brodmann areas $3 \mathrm{~b}$ and1 (Fig. 1). Extracellular small multiunit recordings were made in the $\mathrm{S} 1$, using stimuli as described above for dorsal rootlets. Similar recordings have been described previously (Darian-Smith and Brown, 2000; Darian-Smith, 2004; Darian-Smith and Ciferri, 2005).

Tracing corticospinal terminal fields within the spinal cord. Anterograde tracers BDA (15\% aqueous) or LYD (15\% aqueous) were injected bilaterally into the region of D1-D3 representation in S1 and/or M1 (Fig. 1). All injections, were made using a constant-pressure Hamilton syringe 
(20 $\mu$ l capacity) with a tapered glass micropipette attached (tip diameter, $\leq 30 \mu \mathrm{m}$ ) with 5 min Araldite. Each injection delivered $0.3 \mu \mathrm{l}$ of tracer into the cortex at a $1 \mathrm{~mm}$ depth, and the pipette was kept in position for 1 min before being removed (Fig. 3).

BDA was visualized in axon terminals histochemically, with the attachment of peroxidase (ABC kit; Vector, PK-6100), and the chromagen diaminobenzidine (DAB, Sigma-Aldrich). Tissue sections were cut coronally using a freezing microtome $(50 \mu \mathrm{m})$ and collected and washed in $0.1 \mathrm{M}$ phosphate buffer and $0.1 \%$ Triton (PB-TX), pH 7.4, incubated in ABC for $1 \mathrm{~h}$, rinsed, preincubated in nickel-intensified $\left(0.04 \% \mathrm{NiNH}_{4}\right)$ DAB $(0.05 \% \mathrm{DAB})$ in $0.1 \mathrm{M}$ PB-TX, and finally incubated with the same solution with $0.01 \% \mathrm{H}_{2} \mathrm{O}_{2}$ until the reaction product was clearly visible $(8-10 \mathrm{~min})$.

LYD was visualized with immunohistochemistry. Tissue sections were incubated $\left(4^{\circ} \mathrm{C}\right)$ with primary anti-Lucifer yellow (Invitrogen, catalog \#A5750; RRID:AB_1501344; $48 \mathrm{~h}, 1: 200)$, a secondary antibody, biotinylated anti-rabbit ( $24 \mathrm{~h}$; Vector, BA-1000), and avidin biotin to attach peroxidase (ABC kit; Vector, PK-4000), and reacted with the chromagen DAB $(0.05 \%)$ plus $0.01 \% \mathrm{H}_{2} \mathrm{O}_{2}, 24 \mathrm{~h}$ later. Details have also been reported previously (Darian-Smith et al., 1999, 2013).

Pathway analysis. The spinal cord was sectioned coronally and a series of sections taken for histochemical and immunohistochemical processing to visualize BDA and LYD. An additional series was stained for cresyl violet. All sections were cut $50 \mu \mathrm{m}$ thick and were separated by either $300 \mu \mathrm{m}$ (DRL animals) or $400 \mu \mathrm{m}$ (DRL/ DCL monkeys), with this interval consistent within individual animals.

The distribution territory was defined as the region occupied by axon terminal boutons labeled following the bilateral injection of either LYD or BDA into S1 or M1 cortex (D1-D3 representation). Terminal boutons were mapped using Neurolucida (RRID:nif-0000-10294; MicroBrightField), within a sequential series of coronal sections. Section series were mapped from C1 through T5 (except for DRL animals where sections were mapped from C4 through $\mathrm{T} 1$ ), though statistical analyses were only determined through the lesion zone for sections from C6 through C8 (see Results). A line was drawn to enclose the mapped boutons, and the mean area was calculated (Darian-Smith et al., 2013). Outlying boutons $(<5)$ were not included in the analysis, since they represented $<1 \%$ of the population. For calculating terminal distribution territory volumes, the labeled area was multiplied by the interval distance to the next section, and summed.

The gray matter of spinal cord sections used in the statistical analysis (C6-C8) was divided into three regions, as illustrated in the inset in Figure 9, approximating the dorsal horn (Dorsal), the intermediate zone (Medial), and the ventral horn (Ventral; for comparison, see Kuypers, 1981, description of Rexed's laminae; Morecraft et al., 2013). A macro program was written in the image processing software Fiji (National Institutes of Health, previously ImageJ, RRID:nif-0000-30467), and this was used to partially automate the section division (see Fig. 9). The dorsal region was defined by a line drawn between a point at the base of the midsagittal fissure and the most medial border at the base of the dorsal horn. The centroid was then determined for the remaining area beneath this point and a horizontal line drawn dividing this space using medial and ventral regions (by the macro program). Areas were determined for each of these three regions $\left(D_{1}, M_{1}, V_{1}\right)$, as well as the terminal bouton territory within them $\left(D_{2}, M_{2}, V_{2}\right)$, and terminal territories calculated as percentages for each region $\left(D_{2} / D_{1}, M_{2} / M_{1}, V_{2} / V_{1}\right)$.

Statistical analysis. The number of animals used in our analysis was small, which is part of nonhuman primate research. However, this did not decrease the power of the statistical analysis used, which took sample size, tracer uptake efficacy, and other variables into account (see Materials and Methods; for discussion of analysis, sample size, and power, see Darian-Smith et al., 2013; Hoenig and Heisey, 2001; Levine and Ensom, 2001).

We analyzed the data as a repeated-measures design within monkeys, where the monkey was the subject and individual slices were repeated measures further subdivided into side, region, and projection. This approach gives far higher power than a simple $t$ test approach, providing equivalent power at exponentially lower sample sizes (Grafen and Hails, 2002), not least because it enables us to separate out variance due to individual differences between monkeys, from variance due to biological and technical differences between sections. Such advanced analysis is an essential tool for improving animal welfare in studies of this kind (Russell, 1995).

Our primary variable of interest was the ipsilateral/contralateral staining ratio. When considering the mean ipsilateral/contralateral ratio over multiple repeated measures, this mean is made up of two components. First, the overall mean ratio and, second, the degree to which that ratio is stable when the degree of contralateral staining is particularly high or low. To tease these apart, we used a repeated-measures regression. This enables us to figure the conventional mean ipsilateral/contralateral ratio (by testing the mean ipsilateral staining at the mean level of contralateral staining), controlling for the relative stability of these ratios in the different subareas and treatments (lesion types). Data were analyzed as a Repeated Measures Restricted Maximum Likelihood (REML) mixed model in JMP10 for Windows (SAS). Subject (animal) was nested within treatment (lesion type) as a random blocking factor, and suitable interactions were included to ensure the correct calculation of repeated-measures tests (Newman et al., 1997). Section position was included as a quadratic continuous blocking factor. Subarea (D, M, and V), projection (S1 vs M1), and treatment (lesion type) were included as interactions and nonsignificant interactions were removed to preserve the integrity of testing marginal terms (Grafen and Hails, 2002). Post hoc tests were estimated as REML planned contrasts and, using the Benjamini-Hochberg proce- 


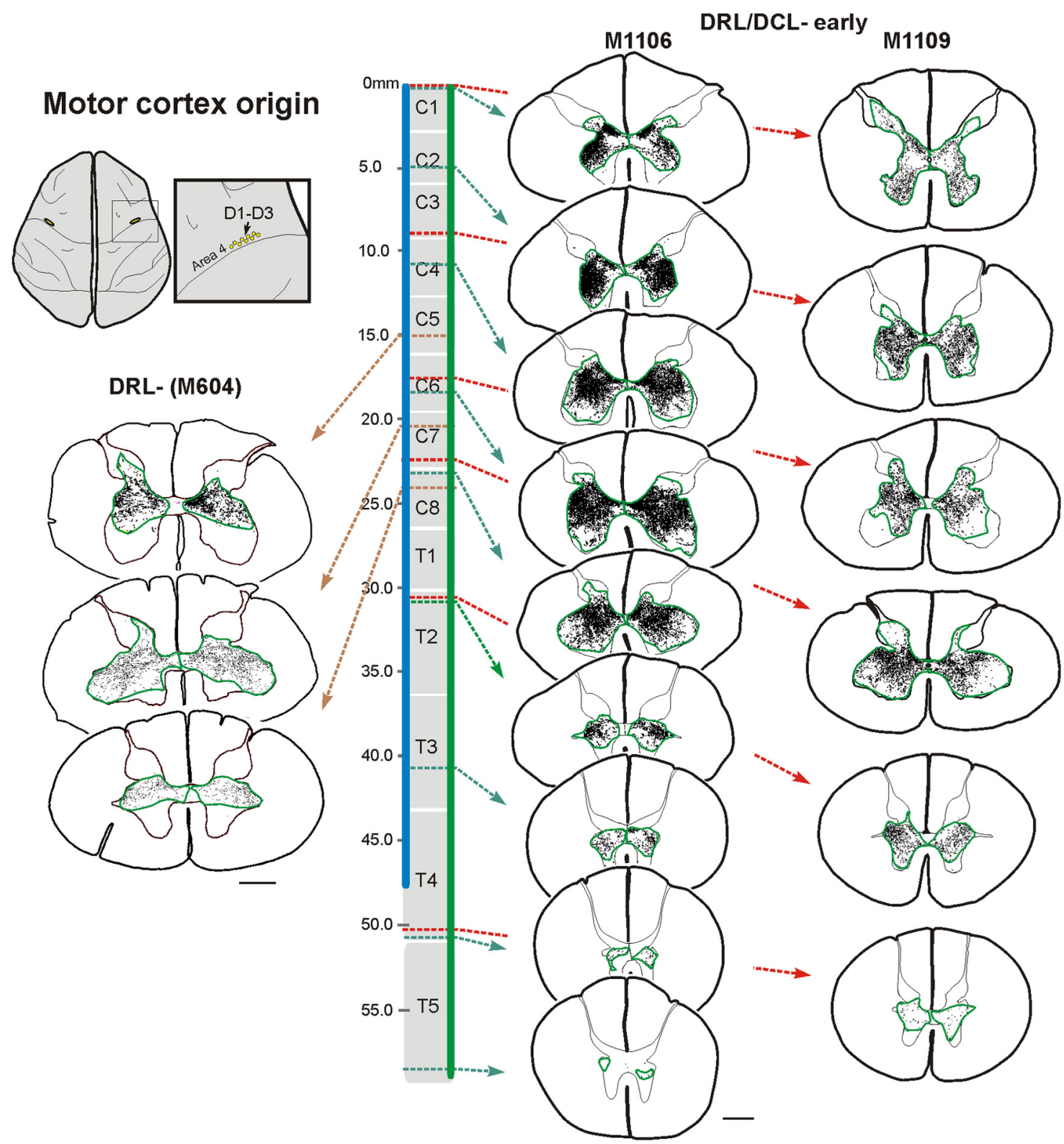

Figure 4. Corticospinal terminal labeling within the spinal segments C1-T5, which originated from the primary motor cortex (D1-D3 representation). Data shown to left (M604) is from a monkey with just a DRL (also reported in Darian-Smith et al., 2013). Data shown on right (M1106 and M1109) is from monkeys that received a combined DRL/DCL lesion at the same time (or "early"; Table 1). The location of each section illustrated is indicated on the schematic of the spinal cord. Blue and green bars indicate the full rostrocaudal extent of terminal labeling for DRL and DRL/DCL early animals, respectively. Green contours demarcate terminal bouton distribution areas. Note that terminal labeling extended $\sim 15 \mathrm{~mm}$ farther caudally in DRL/DCL monkeys compared with the DRL monkey. Lesion is on the left side. Scale bar, $1 \mathrm{~mm}$.

dure, corrected for multiple-testing false-discovery rates (Benjamini and Yekutieli, 2001). The assumptions of mixed models (linearity, homogeneity of variance, and normality of error) were confirmed post hoc (Grafen and Hails, 2002).

All figures were assembled and created in CorelDraw X4, and photomicrographs adjusted for sharpness, contrast, and brightness within Adobe PhotoShop (CS5).

\section{Results}

Corticospinal projections were analyzed in six monkeys (Table 1). Injections were always made bilaterally in the region of D1-D3 representation in S1, which was identified electrophysiologically (Fig. 1). M1 injections were made opposite the S1 sites on the opposite side of the central sulcus.

To ensure that labeling did not occur in response to changes in membrane uptake properties following injury, BDA and LYD were used interchangeably in different animals in the S1 and M1 cortex. Our statistical analysis controlled for confounds due to tracer and confirmed that there were no significant tracer-related effects in the data (Darian-Smith et al., 2013). Tracer-uptake anomalies were therefore not considered a concern in the present study.

\section{Defining cortical injection sites and lesion extent}

Injections within the cortex were examined through a series of sections within the region of hand representation in the sensorimotor cortex. Injection sites did not extend beyond the cortical gray matter or across the central sulcus, which could have compromised the data (Fig. 3). Though the boundary of the injection sites was not always sharply delineated (making volume analysis impossible), injections were always matched precisely for location (i.e., within the region of D1-D3 representation, determined 
electrophysiologically; Fig. 1), number, and tracer volume injected. Injection sites were typically localized to cytoarchitectonic areas $3 \mathrm{~b}$ and 1 , as defined by adjacent Nissl-stained sections, though some involvement of rostral area 2 cannot be ruled out. Figure 3 shows an example of injection sites from adjacent sections and both hemispheres in M1109. There were no differences in the cortical injection extent between hemispheres in any of the monkeys that could have accounted for the differences in the terminal labeling observed and described below.

Figure 2 shows that the DCL in each of the DRL/DCL monkeys only involved the cuneate fasciculus in M1107 and M1106 (as far as could be determined from lesion reconstruction), with some additional gray matter involvement in M1109 and M1110. Thus the central injury may have involved cutaneous afferents from the remaining digits (D4 and D5) or forearm that ascend in the dorsal column, but primary afferents for the rest of the body and hindlimb would not have been affected. Importantly, the spinothalamic projection from D4 and D5 was not cut by the lesion, and the central component of the DRL/DCL monkeys largely affected primary afferents already involved by the DRL, as well as secondary neurons originating below the DCL, and any additional fibers traversing this tract. The spinothalamic pathway mainly transmits information from $A \delta$ and $C$ receptors (or pain and temperature information), but past studies suggest that this pathway may also encode crude cutaneous and proprioceptive information, either via $\mathrm{A} \delta$ and $\mathrm{C}$ fibers or via spared cutaneous afferents that branch and synapse on spinothalamic afferents within the dorsal horn. Thus D4 and D5 were only partially deafferented in monkeys receiving a combined DRL/DCL lesion, which is unlikely to have translated to a detectable behavioral deficit in these digits. We propose that the addition of the DCL in the DRL/DCL does not significantly affect hand function beyond that already observed following a DRL (DarianSmith and Ciferri, 2005). Behavioral data obtained from one DRL/DCL monkey, M1106 (not reported here), supports this, since we observed little difference in the deficit or recovery of hand function (tested for D1-D3) in this monkey compared with a DRL alone. Further behavioral data, however, are needed to fully test this.

\section{Analysis of terminal territories}

Though the terminal bouton distribution territory was mapped in sections at regular intervals (from $\mathrm{C} 1$ to $\mathrm{T} 5$ for DRL/DCL animals and from C5 to T1 in DRL monkeys), our statistical analysis was conducted only on sections spanning C6-C8. The reason for this was as follows. DCLs were made level with the rostral DRL border, which was either in caudal C5 (M1109,

\section{Motor cortex origin} DRL/DCL- late
M1107

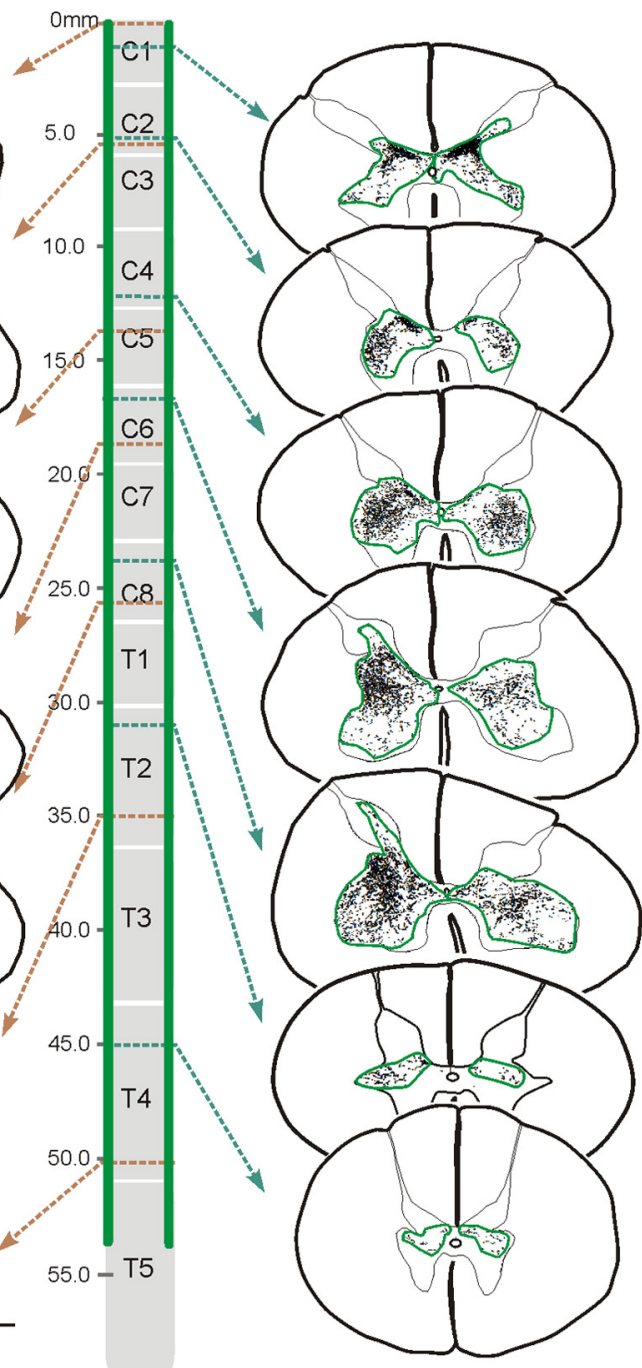

Figure 5. Corticospinal terminal labeling within the spinal segments $\mathrm{C} 1-\mathrm{T5}$, which originated from the primary motor cortex (D1-D3 representation) from monkeys that received a combined DRL/DCL lesion, where the DCL component was made several months following the initial DRL lesion (DRL/DCL late; Table 1). The location of each section and rostrocaudal extent of labeling is indicated with green bars on spinal cord schematic. Conventions as in Figure 4. Lesion is on the left side. Scale bar, $1 \mathrm{~mm}$.

M1110) or rostral C6 (M1106, M1107). C6-C8 was therefore the region of the cord where most of the D1-D3 afferent inputs were mapped (electrophysiologically) and cut when making the DRL during the initial surgery. This is where the greatest changes in circuitry were likely to be occurring and where we observed the greatest differences in the CST labeling patterns on the two sides of the cord. We were able to determine the boundaries of this region very accurately during the initial surgery, and again in the postmortem spinal cord dissection, where the segments, cut rootlets, and dorsal root entry of these to the cord were clearly visible. In sections both rostral and caudal to this region, terminal distribution territories were typically similar between the two sides of the cord, regardless of the animal or lesion type (Figs. 4-7).

\section{Corticospinal projection patterns}

The ipsilateral/contralateral staining ratio differed between M1 and $\mathrm{S} 1$, between the different subareas, and between the treatments (treatment-subarea-pathway interaction: $F_{(4,10.71)}=$ 


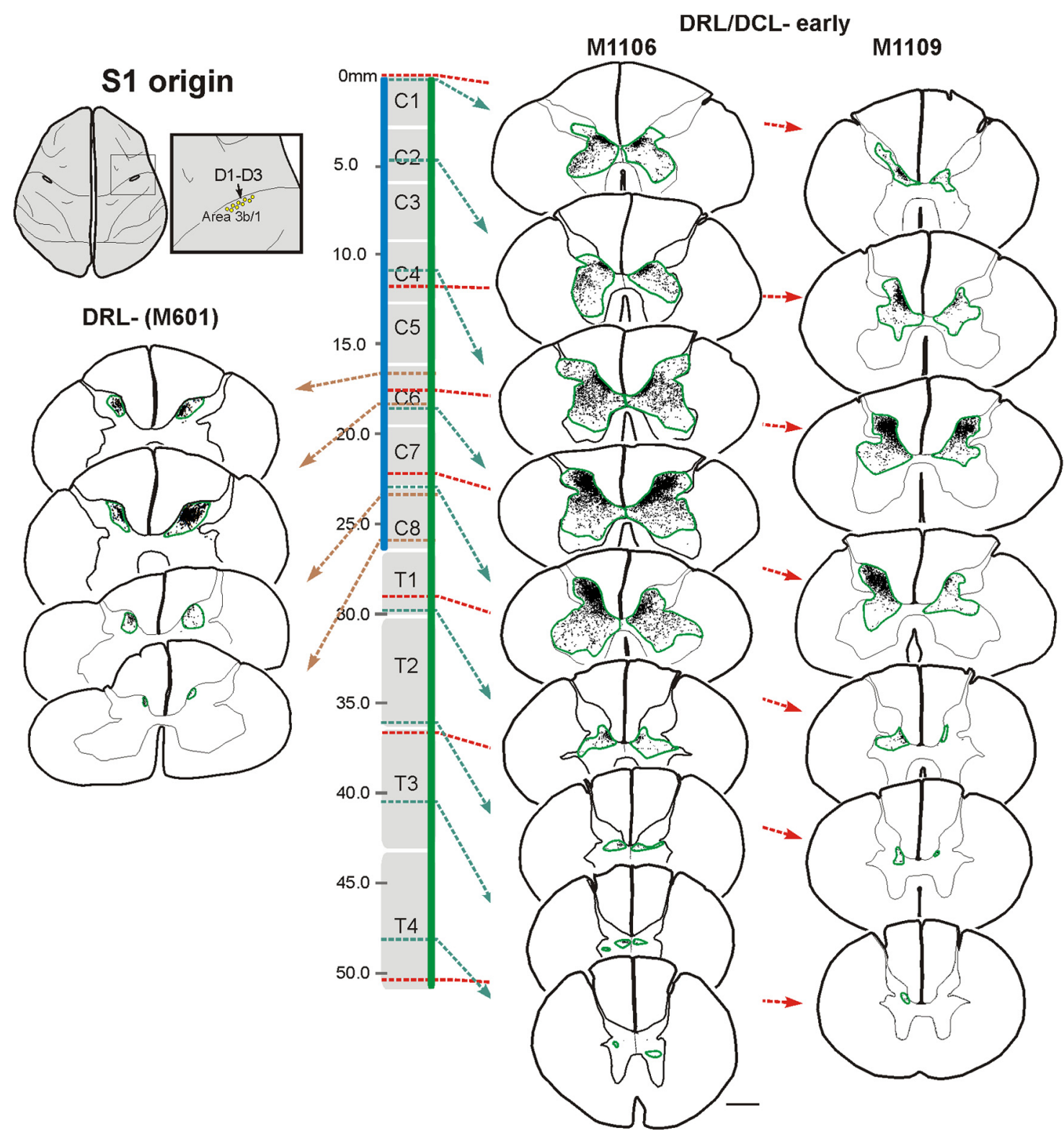

Figure 6. Corticospinal terminal labeling within the spinal segments (1-T4, which originated from the primary somatosensory cortex (D1-D3 representation). Data shown to left (M601) is from a monkey with just a DRL (also reported in Darian-Smith et al., 2013). Data shown on right (M1106 and M1109) are from monkeys that received a combined DRL/DCL lesion at the same time (or "early"; Table 1). Conventions as in Figure 4. Note that terminal labeling extended $\sim 25 \mathrm{~mm}$ farther caudally in DRL/DCL monkeys compared with the monkey receiving a DRL alone. Lesion is on the left side. Scale bar, $1 \mathrm{~mm}$.

5.6784; $p=0.0105$; see Fig. 10 ). The following sections explore this result and describe the pattern of terminal labeling within the spinal cord for M1 and S1 CST subcomponents.

\section{Motor cortex}

Following a DRL

The data for the DRL monkeys used in this study (M604 and M601) were reported in detail in our recent paper (Darian-Smith et al., 2013). Briefly, and as described in that study, the M1 CST in DRL monkeys responded by sprouting into the dorsal horn in the lesion zone on the side of the lesion. This was observed in three of four monkeys, with the mean terminal territory $>110 \%$ of the contralateral side. This is summarized for M604 in Figures 3 and 10. Note that following a DRL alone, the pattern of labeling on the side contralateral to the lesion was equivalent to control nonle- sioned animals as previously described (Galea and Darian-Smith, 1997b; Rosenzweig et al., 2010; Morecraft et al., 2013).

\section{Following a $D R L / D C L$}

The response from the M1 CST following a DRL/DCL was greater than was observed following the DRL alone (Figs. 4, 5; see Fig. 11). In all four monkeys (regardless of whether the DCL component was made early or late) we observed the same extension into the dorsal horn as was observed following a DRL alone. In addition, we also consistently observed more ventral terminal labeling (compared with DRL animals) within the lesion zone, both throughout the ventral horn and bilaterally (Figs. 4, 5; see Fig. 11).

Figure 9 shows the mean histogram profiles of terminal territories (C6-C8) as a percentage of gray matter for the dorsal, medial, and ventral regions of the spinal cord. Each of the lesion 
groups and ipsilateral versus contralateral sides of the cord relative to the lesion are shown for both the M1 and S1 CST terminal territories. Not surprisingly, when analyzed as ipsilateral/contralateral ratios (see Fig. 10), data show significantly different overall profiles of terminal labeling for the DRL versus the DRL/DCL animals and for M1 versus S1 CSTs.

For the M1 CST, the pattern of ipsilateral/contralateral ratios in the different subareas was significantly different in each treatment (within M1, subareatreatment interaction: $F_{(8,16.23)}=9.5265$; $p<0.0001)$. Further post hoc tests of this result showed that staining in the terminal territory was significantly greater following a DRL/DCL (mean of both treatments) versus a DRL in the ventral subarea $\left(F_{(1,18.28)}=16.6763 ; p=0.0007\right)$ and the medial subarea $\left(F_{(1,27.17)}=\right.$ $12.3093 ; p=0.0016$ ), whereas the dorsal region did not differ between treatments $\left(F_{(1,16.87)}=0.0873 ; p=0.7713\right)$.

Terminal labeling was also observed bilaterally from $\mathrm{C} 1$ through $\mathrm{T} 5$ following the combined lesion (Figs. 4, 5). Terminal labeling in T5 indicated caudal sprouting for $\sim 10-15 \mathrm{~mm}$ beyond the ( $\sim 45 \mathrm{~mm}$ C1-T4; Fig. 4) rostrocaudal territory observed in monkeys with a DRL alone.

As a coarse measure of comparison, absolute terminal territory volumes were also calculated through segments C6-C8 in all monkeys. The overall terminal territory volume for M1 DRL monkeys was found to be a little more than half that calculated for DRL/DCL monkeys (mean, $16.96 \mathrm{~mm}^{3}$; $\mathrm{SD}, \pm 4.8$ ) versus $28.89 \mathrm{~mm}^{3}$ $( \pm 3.7)$ ipsilaterally, and $14.66 \mathrm{~mm}^{3}$ $( \pm 6.0)$ versus $24.22 \mathrm{~mm}^{3}$ ( \pm 2.6$)$ contralaterally, in DRLs versus DRL/DCLs respectively).

\section{Somatosensory cortex}

Following a DRL

As we have previously reported (Darian-Smith et al., 2013), the S1 CST terminal territory was reduced to $<60 \%$ on the side of the lesion, compared with the contralateral side of the cord. This was in contrast to the M1 CST response in the same DRL animals, and suggests a reduced role for this pathway in circuit reorganization following a DRL alone. Figure 6 shows four sections from M601, and Figure 9 summarizes the reduced S1 CST response in the DRL animals (Darian-Smith et al., 2013).

Following a DRL/DCL

Surprisingly, and counter to our observations following a DRL alone, the S1 CST response was bilateral and dramatic following the combined DRL/DCL. The overall terminal territory volume for the S1 CST in DRL/DCLs (C6-C8) was four times that calculated in DRL animals on the ipsilateral side of the cord (mean, $4.84 \mathrm{~mm}^{3}$; SD, \pm 2.3 ; vs mean, $20.95 \mathrm{~mm}^{3}$; SD, \pm 4.6 ) and almost three times the DRL volume on the contralateral side (mean, 6.96 $\mathrm{mm}^{3}$; $\mathrm{SD}, \pm 2.9$; vs mean, $17.94 \mathrm{~mm}^{3}$; $\left.\mathrm{SD}, \pm 5.6\right)$.

\section{S1 origin}

DRL/DCL- late M1107

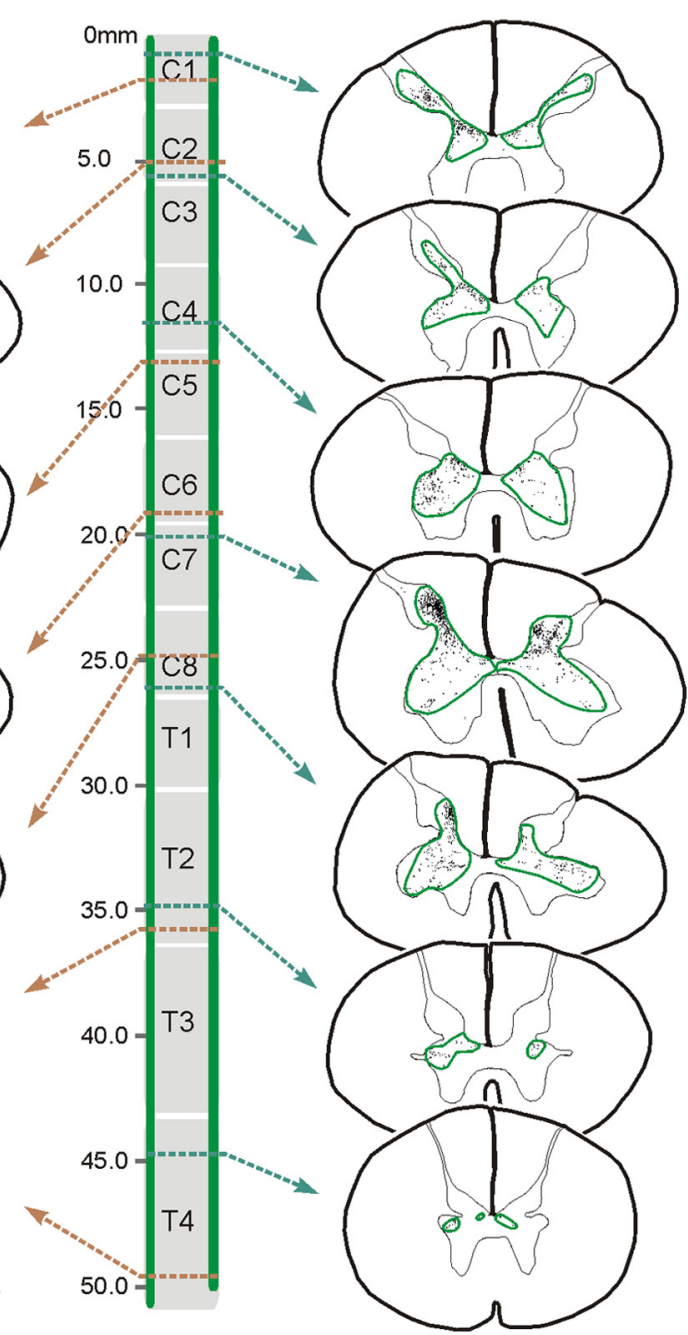

Figure 7. Corticospinal terminal labeling within the spinal segments C1-T4, which originated from the S1 cortex (D1-D3 representation) from monkeys that received a combined DRL/DCL lesion, where the DCL component was made several months rostrocaudal extent of labeling is indicated with green bars on spinal cord schematic. Consistent with all DRL/DCL monkeys, terminal labeling was observed caudally to caudal T4. Lesion is on the left side. Scale bar, $1 \mathrm{~mm}$.
Terminal labeling patterns are shown for all four monkeys in Figures 6 and 7, and summarized in Figures 9-11. In all four DRL/DCL monkeys, the S1 CST terminal territory extended ventrally into the medial region or intermediate zone (most obvious within segments C6-C8) and, in three of the four monkeys, this extension was observed into the ventral horn. The absence of this extension into the ventral horn in M1109 (Fig. 6) may simply reflect interanimal variability, since this was not the result of this monkey having fewer cortical injections relative to the other monkeys (Fig. 1).

For the S1 CST, the pattern of ipsilateral/contralateral ratios in the different subareas was significantly different in each treatment (within S1, subarea-treatment interaction: $F_{(8,14.32)}=10.0787 ; p=$ $0.0001)$. Further post hoc tests of this result showed that staining in the terminal territory was significantly greater following a DRL/DCL (mean of both treatments) versus a DRL in the dorsal subarea $\left(F_{(1,13.75)}=17.5707 ; p=0.0009\right)$ and the medial subarea $\left(F_{(1,16.3)}=\right.$ $61.9035 ; p<0.0001)$, whereas the ventral region did not differ between treatments $\left(F_{(1,20.38)}=2.0692 ; p=0.1655\right)$. 

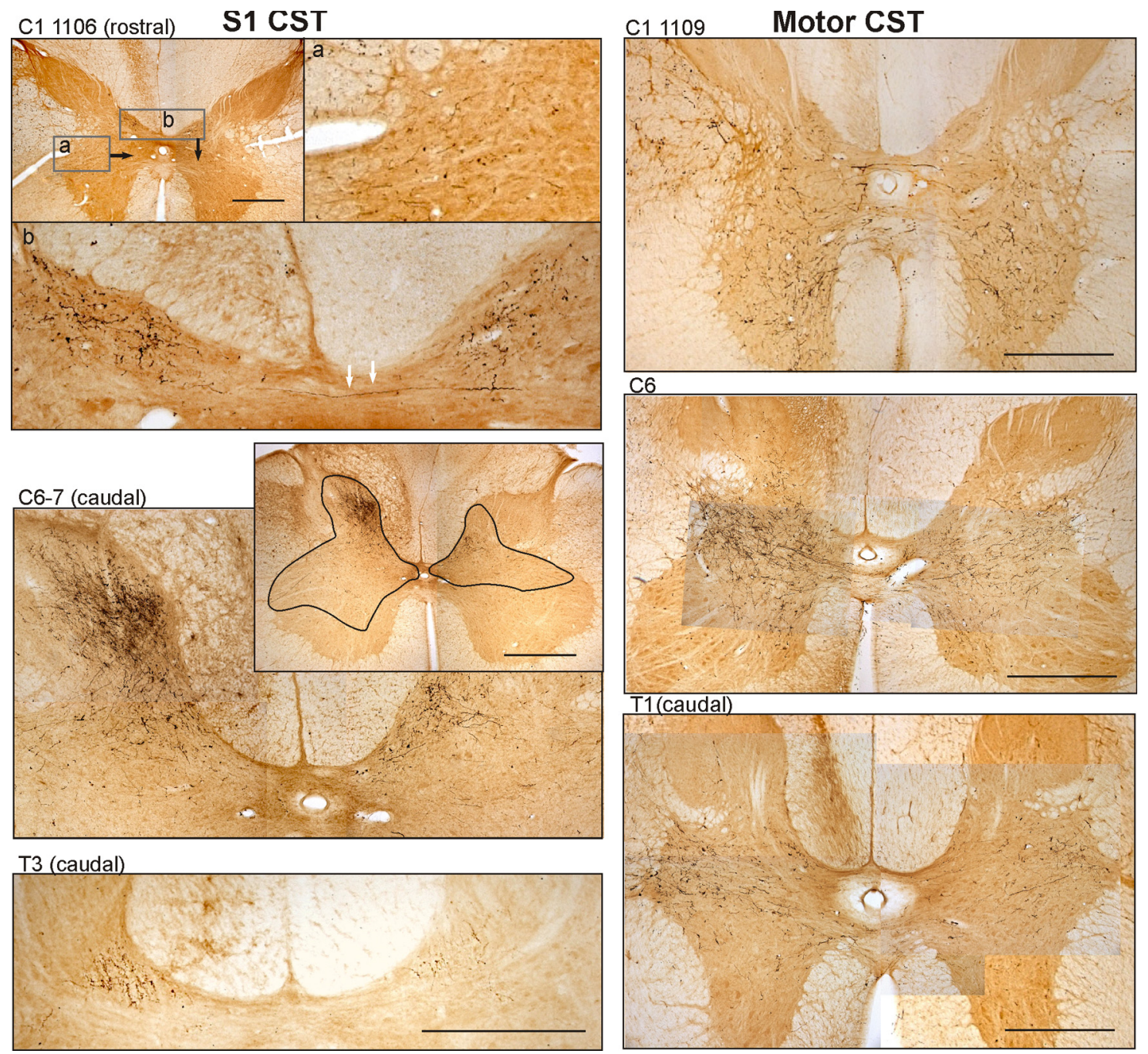

Figure 8. Photomicrographs (including montages) of labeling in M1106 and M1109 showing terminal distributions following a DRL/DCL, within cervical and thoracic segments. Examples are shown from the $\mathrm{S1}$ (left column) and primary motor (right column) corticospinal tracts. Lesion side is on the left for all images. Scale bar, $1 \mathrm{~mm}$.

In addition, terminal labeling consistently extended rostrocaudally in DRL/DCL monkeys from C1 through T4 (Fig. 8, label in T3; see Fig. 11). The caudal extension (i.e., sprouting) of terminal labeling was bilateral for the S1 CST in all four DRL/DCL monkeys, and extended for $\sim 4$ segments beyond what we observed for DRL animals. Terminal labeling was located within the intermediate zone within thoracic segments and gradually diminished caudally, though not always equally on both sides for M1109 and M1107 (Figs. 6, 7, thoracic segments). The extension observed in these animals was twice the total rostrocaudal spread ( $\sim 50 \mathrm{~mm}$ total vs $\sim 25 \mathrm{~mm}$ ) observed in the DRL monkeys. The assumption was made that terminal labeling extended rostrally to $\mathrm{C} 1$ in the DRL animals, as has been described in the literature (Kuypers, 1960; Galea and Darian-Smith, 1997b; Lee and Kim, 2012).

It should also be noted that while we did not include data from C1-C5 in our statistical analyses, Figures 6 and 7 show bilateral labeling in the intermediate and ventral horns through the most rostral part of $\mathrm{C} 1$ in all DRL/DCL animals. In addition, terminal labeling was observed in the dorsal horn on the side of the lesion in rostral cervical segments (in three of four DRL/DCL monkeys), which was not observed contralaterally (Figs. 6, 7; see Fig. 11). Comparable data were not obtained from $\mathrm{C} 1-\mathrm{C} 3$ in DRL monkeys (for S1 CSTs), so it is not known if this labeling was atypical. Figure 11 summarizes the extensive S1 CST sprouting induced following a DRL/DCL compared with a DRL alone.

\section{Comparing early and late DRL/DCL data}

An important observation in the present study was that M1 and S1 CST responses were similar in all four DRL/DCL monkeys even though two monkeys (M1110 and M1107) received the central DCL 4 months following the initial DRL lesion. We performed a series of post hoc tests to assess differences between DRL/DCL early and DRL/DCL late animals, including testing for mean overall differences (e.g., is the mean value for all S1 projections different between early and late animals?) and for betweenregion differences (e.g., whether the difference between medial and ventral M1 scores differed between treatments). None of these tests yielded a significant result. Though the DCL in M1109 and M1110 involved some gray matter, this did not appear to affect the terminal labeling patterns.

\section{Discussion}

We have shown that the S1 and M1 CSTs respond very differently following a DRL (peripheral) versus a DRL/DCL (peripheral plus central) in monkeys. With a DRL alone, input to the cervical cord from the S1 cortex diminished on the side of the lesion to $\sim 60 \%$ 


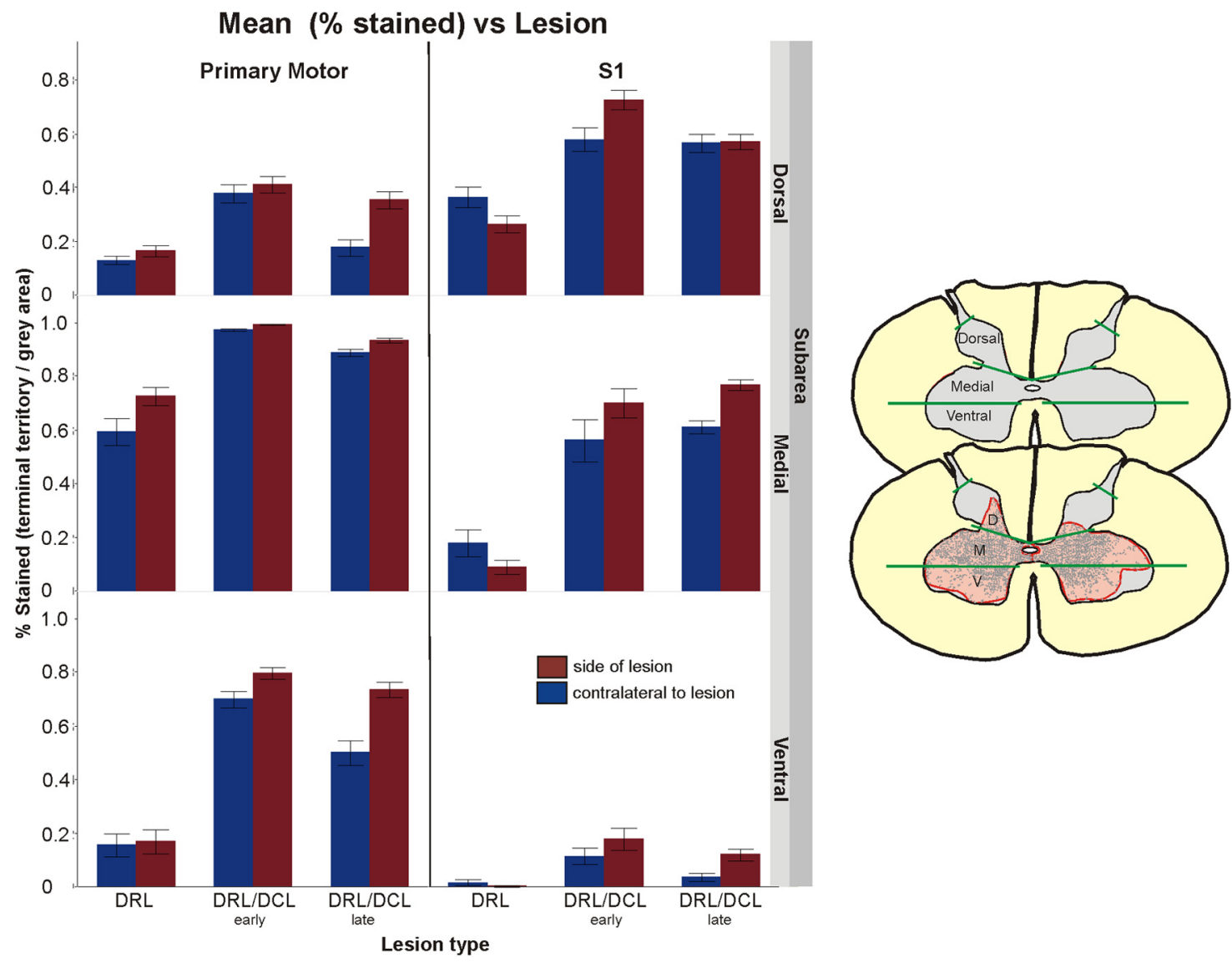

Figure 9. Histogram showing the data used in the statistical analysis in this study. The CST terminal territory is shown as the percentage of gray matter in the dorsal, medial, and ventral subdivisions. Mean percentages are given for both sides of the cervical cord and for M1 and S1 CSTs. Subdivisions D, M, and V are defined for typical section at right (see text for details). Data were collected for C6 - C8. Error bars indicate SEM. Most of the major findings of this study are apparent here. For example, in medial S1 there was significantly more labeling following a DRL/DCL versus a DRL, and in ventral M1, there was significantly more labeling following a DRL/DCL versus a DRL.

of the original distribution territory and the M1 CST remained intact or increased (Darian-Smith et al., 2013). In contrast, with the addition of a central DCL (C5/ C6), there was a massive and bilateral expansion of terminal labeling during the following months from both the S1 and M1 CSTs. This response was similar in all monkeys receiving a combined DRL/ DCL, regardless of whether the DCL was made at the same time or 4 months after the DRL. The pattern of terminal labeling following the DRL/DCL was also quite different for the S1 and M1 CST subcomponents.

The degree of sprouting observed in our monkeys following the DRL/DCL is unprecedented and, from the reorganized S1 cortex, particularly dramatic. This suggests that the S1 CST response may be as important an indicator of recovery as the M1 CST, at least in primates.

In discussing our findings, certain caveats should be kept in mind. Since the same tracer was injected into both hemispheres, it was not possible to determine the hemisphere of origin of the CST sprouting. This may be important for the M1 CST response, where many fibers cross the midline before and after spinal in-

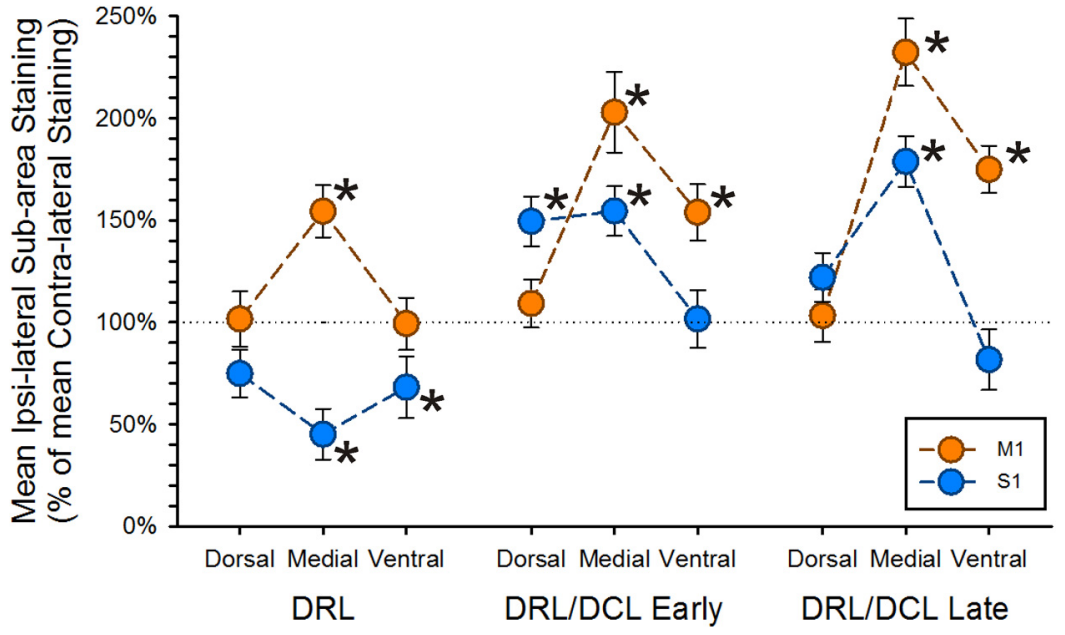

Figure 10. Summary of the CST terminal labeling patterns from $S 1$ and $M 1$ following a DRL compared with a DRL/DCL, showing the ipsilateral/contralateral distribution territory ratio. Values $>100 \%$ indicate greater relative staining on the side ipsilateral to the lesion. Values $<100 \%$ indicate greater relative staining contralaterally. Asterisks indicate values significantly different from $100 \%$.

jury. However, crossover fibers are unlikely to play a significant role in the S1 CST response (at least for hand), since they are not present in normal and DRL animals (Darian-Smith et al., 2013), and are almost nonexistent following the DRL/DCLs (i.e., we observed only 1-2 per section in C1-C3). In addition, terminal bouton density was not determined in this study, since different 

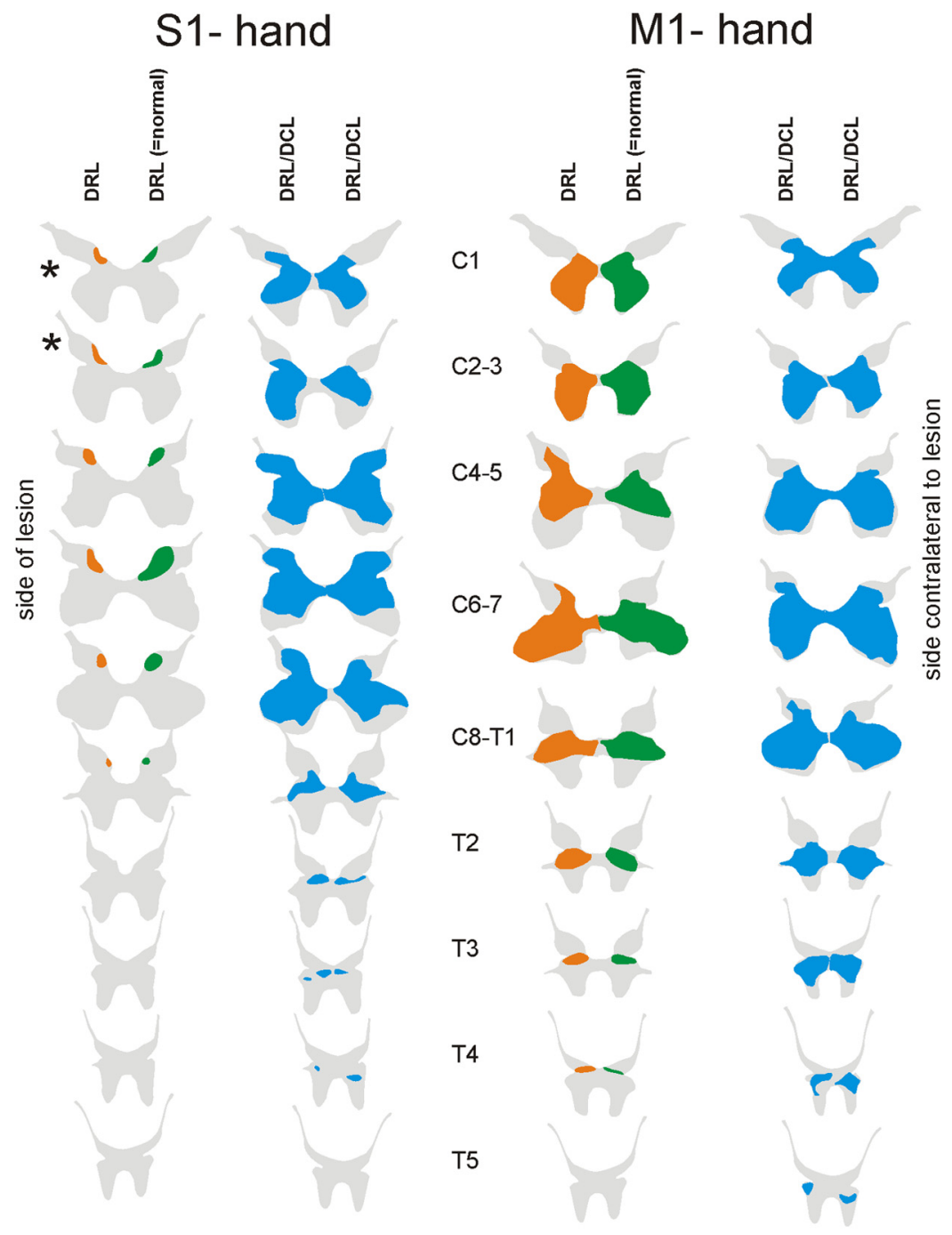

Figure 11. Summary of the CST terminal labeling patterns from $S 1$ and $M 1$ following a DRL compared with a DRL/DCL. Columns with green shading show terminal labeling patterns contralateral to the DRL, which are equivalent to nonlesioned terminal distributions. Orange shading shows terminal labeling patterns ipsilateral to the DRL, and blue shows terminal labeling patterns on both sides following the combined DRL/DCL. Major changes are clear. First, the S1 CST plays little part following a DRL, but expands dramatically and bilaterally following a DRL/DCL, with a caudal extension of terminals from C8/T1 to caudal T4. For the motor CST, terminal sprouting extends into the dorsal horn on the side of the lesion following a DRL. Following a DRL/DCL, the terminal sprouting extends bilaterally into the ventral horn, and caudally for an additional segment into T5. Asterisks indicate data not collected in the current study, and predicted from published findings (Kuypers, 1960; Galea and Darian-Smith, 1997a; Lee and Kim, 2012) and more caudal patterns.

tracer injection series were made into the two hemispheres, and a bouton density comparison between sides would be difficult to interpret (Darian-Smith et al., 2013). Having said this, we consistently noted more terminal boutons ipsilateral to the lesion (C5-C8) in the DRL/DCL animals, which is opposite to our observations in DRL monkeys (Darian-Smith et al., 2013).

Interestingly, animals in the early DRL/DCL group and animals in the late DRL/DCL group did not differ statistically in their response (Figs. 9-11), which raises an important clinical question, especially for chronic patients. Could an even smaller central spinal injury awaken and augment the formation of new connections and induce additional recovery many months (or even years) following a disabling rhizotomy or brachial plexus injury? This is a subject for future study.

So what fundamentally changes with the inclusion of a central (dorsal cuneate fasciculus) lesion to induce such a dramatic re- sponse from both M1 and (especially) S1 CSTs? The answer almost certainly lies with immune response and/or the location of the DCL component of the DRL/DCL.

In contrast to peripheral injuries, CNS injuries result in an enhanced immune response that involves a cascade of acute to chronic events, a massive astrocytic and microglial proliferation, and the eventual formation of a cellular scar (Fitch et al., 1999; Darian-Smith, 2009; Rolls et al., 2009; Karimi-Abdolrezaee and Billakanti, 2012). The cellular and molecular environment changes dramatically at the site of injury (Yang et al., 2006) and well beyond, and, while many factors associated with this altered environment are essential for protection and repair of surviving neurons (David et al., 2012), others can inhibit CST sprouting (e.g., chondroitin sulfate proteoglycans; Fitch and Silver, 2008; Rhodes and Fawcett, 2004; ephrins; Miranda et al., 1999; Fabes et al., 2006). While our understanding of the immune response over time following different spinal injuries remains poor (particularly in primates), determining the CST response following a DCL injury alone will help test its importance. If similar M1 and S1 CST sprouting is observed, then clearly the central injury and changing CNS environment are key to our understanding of the mechanisms involved. If, however, terminal sprouting from the S1 and M1 CSTs is not observed following a DCL alone, then there may be an interaction between the peripheral and central injuries that will require further investigation.

Another explanation for the different CST sprouting response following the two lesions is that different circuitry was affected by each. A DRL alone removes all primary afferent input from the affected digits but leaves the central circuitry intact. Though the DRL/DCL largely cuts the same primary afferents already cut by the DRL, it also involves more caudal, primary afferents of the hand. The spinothalamic input remains intact below the dorsal rhizotomy, as do all descending projections (i.e., bulbospinal, reticulospinal, rubrospinal, etc.), so D4-D5 are only partially deafferented. Most sensory reafference, which requires input from the periphery, is lost for D1-D3 in both lesions. However, the central cuneate fasciculus lesion in the DRL/DCL blocks additional intraspinal connections not cut by the DRL alone, and this may contribute to the different CST responses following the two different lesion models.

From where do these atypical projections originate? In normal macaque monkeys, as much as $98 \%$ of the motor "hand" CST projection arises from the contralateral cortex and descends in the dorsolateral CST (dlCST), with 2\% descending in the ipsilateral dlCST (Morecraft et al., 2013). M1 CST axons are also known to send collaterals to the contralateral side of the cord. Previous 
studies in macaque monkeys that looked at the M1 CST response following spinal hemisection suggest that these collaterals play a role in postinjury sprouting and the recovery process (Galea and Darian-Smith, 1997b; Rosenzweig et al., 2009). In keeping with these earlier reports, we also clearly observed M1 CST axons crossing the midline in cervical and thoracic segments following DRLs and DRL/DCLs (Fig. 8). However, their hemispheric origin and the importance of their contribution to bilateral sprouting and postinjury recovery remains unclear.

In nonlesioned monkeys, the S1 CST terminates in a confined region of the dorsal horn and there is no evidence of crossover collateral branching. Following a DRL/DCL in this study, a few labeled S1 CST axons were observed in the midline in C1-C3 (Fig. 8), but none were observed below these segments in either the DRL/DCL or DRL monkeys, so S1 CST crossover axons are unlikely to play a significant role in reorganization after injury. Given this, what could be driving the bilateral response following the combined DRL/DCL? One possibility is that callosal connections to the ipsilateral hemisphere drive the CST terminal sprouting in the spinal cord contralateral to the lesion. While callosal projections connect the hand region of M1 (Brodmann area 4) interhemispherically in macaques, they are surprisingly sparse in the hand region in S1 areas 3b and 1 (Killackey et al., 1983; Iwamura, 2000). They are, however, reasonably dense for areas 2 and 5 , where bilateral RFs are commonly found (Iwamura, 2000). It is therefore feasible that the partially deafferented $S 1$ hand region receives callosal input either directly from area 2, or indirectly (i.e., via cortococortical connections), and that callosal input helps drive M1 and S1 CST sprouting in the contralateral cord.

Surprisingly, following a DRL/DCL, the M1 and S1 CST terminal distribution extended caudally well into the thoracic cord. For the S1 CST, terminal labeling extended as far as T4 (i.e., four segments) or double the distance (i.e., a total of 50 vs $25 \mathrm{~mm}$ ) observed in normal monkeys (Galea and Darian-Smith, 1997b) or those with a DRL alone (Darian-Smith et al., 2013). For the M1 CST, terminal bouton labeling was observed as far as rostral T6 (M1106), or an additional segment beyond what we observed in DRL monkeys. The role of these caudal connections is not known, but they may enhance intraspinal and propriospinal connectivity supporting hand-trunk coordination following injury.

At the rostral pole of the cervical cord, dense terminal labeling was also observed (for both S1 and M1 CSTs in DRL/DCL monkeys) in the internal basilar nucleus close to the midline (Figs. $4-7,10)$. Though little is known about the role of this nucleus, it is continuous with the cuneate nucleus (Cliffer and Giesler, 1989). It receives input from ulnar and radial nerves (LaMotte et al., 1991) and from the cervical enlargement (Cliffer and Giesler, 1989). It also receives CST input from the sensorimotor cortex (Kuypers, 1960; Antal, 1984; Bortoff and Strick, 1993; Valtschanoff et al., 1993). It seems likely that this region may also contribute to postinjury reorganization and recovery.

Our results raise important questions. What spinal neurons do newly formed terminals synapse with and are they functional? What is the time course for their formation? How does the cellular and molecular microenvironment change over time? And how do our findings correlate with behavioral recovery?

Our findings indicate that the role of the S1 CST in reorganization following a DRL/DCL may be as important as the M1 CST in the recovery process following a central spinal injury. They also suggest that the S1 CST may present an important additional biomarker of recovery following spinal injury. Certainly, the role of the CST subcomponents in recovery following spinal injury warrants further study, and should be considered in the interpretation and design of future experiments.

\section{References}

Antal M (1984) Termination areas of corticobulbar and corticospinal fibres in the rat. J Hirnforsch 25:647-659. Medline

Benjamini Y, Yekutieli D (2001) The control of the false discovery rate in multiple testing under dependency. Ann Stat 29:1165-1168. CrossRef

Bortoff GA, Strick PL (1993) Corticospinal terminations in two new-world primates: further evidence that corticomotoneuronal connections provide part of the neural substrate for manual dexterity. J Neurosci 13:51055118. Medline

Brus-Ramer M, Carmel JB, Chakrabarty S, Martin JH (2007) Electrical stimulation of spared corticospinal axons augments connections with ipsilateral spinal motor circuits after injury. J Neurosci 27:13793-13801. CrossRef Medline

Catsman-Berrevoets CE, Kuypers HG (1976) Cells of origin of cortical projections to dorsal column nuclei, spinal cord and bulbar medial reticular formation in the rhesus monkey. Neurosci Lett 3:245-252. CrossRef Medline

Cliffer KD, Giesler GJ Jr (1989) Postsynaptic dorsal column pathway of the rat. III. Distribution of ascending afferent fibers. J Neurosci 9:3146-3168. Medline

Darian-Smith C (2004) Primary afferent terminal sprouting after a cervical dorsal rootlet section in the macaque monkey. J Comp Neurol 470:134150. CrossRef Medline

Darian-Smith C (2009) Synaptic plasticity, neurogenesis, and functional recovery after spinal cord injury. Neuroscientist 15:149-165. CrossRef Medline

Darian-Smith C, Brown S (2000) Functional changes at periphery and cortex following dorsal root lesions in adult monkeys. Nat Neurosci 3:476481. CrossRef Medline

Darian-Smith C, Ciferri MM (2005) Loss and recovery of voluntary hand movements in the macaque following a cervical dorsal rhizotomy. J Comp Neurol 491:27-45. CrossRef Medline

Darian-Smith C, Tan A, Edwards S (1999) Comparing thalamocortical and corticothalamic microstructure and spatial reciprocity in the macaque ventral posterolateral nucleus (VPLc) and medial pulvinar. J Comp Neurol 410:211-234. CrossRef Medline

Darian-Smith C, Hopkins S, Ralston HJ 3rd (2010) Changes in synaptic populations in the spinal dorsal horn following a dorsal rhizotomy in the monkey. J Comp Neurol 518:103-117. CrossRef Medline

Darian-Smith C, Lilak A, Alarcón C (2013) Corticospinal sprouting occurs selectively following dorsal rhizotomy in the macaque monkey. J Comp Neurol 521:2359-2372. CrossRef Medline

Darian-Smith I, Galea MP, Darian-Smith C, Sugitani M, Tan A, Burman K (1996) The anatomy of manual dexterity. The new connectivity of the primate sensorimotor thalamus and cerebral cortex. Adv Anat Embryol Cell Biol 133:1-140. CrossRef Medline

David S, Lópes-Vales R, Wee Yong V (2012) Harmful and beneficial effects of inflammation after spinal cord injury: potential therapeutic implications. Handb Clin Neurol 109:485-502. CrossRef Medline

Dum RP, Strick PL (1991) The origin of corticospinal projections from the premotor areas in the frontal lobe. J Neurosci 11:667-689. Medline

Fabes J, Anderson P, Yáñez-Muñoz RJ, Thrasher A, Brennan C, Bolsover S (2006) Accumulation of the inhibitory receptor EphA4 may prevent regeneration of corticospinal tract axons following lesion. Eur J Neurosci 23:1721-1730. CrossRef Medline

Fitch MT, Silver J (2008) CNS injury, glial scars, and inflammation: inhibitory extracellular matrices and regeneration failure. Exp Neurol 209:294301. CrossRef Medline

Fitch MT, Doller C, Combs CK, Landreth GE, Silver J (1999) Cellular and molecular mechanisms of glial scarring and progressive cavitation: in vivo and in vitro analysis of inflammation-induced secondary injury after CNS trauma. J Neurosci 19:8182-8198. Medline

Galea MP, Darian-Smith I (1994) Multiple corticospinal neuron populations in the macaque monkey are specified by their unique cortical origins, spinal terminations, and connections. Cereb Cortex 4:166-194. CrossRef Medline

Galea MP, Darian-Smith I (1997a) Manual dexterity and corticospinal connectivity following unilateral section of the cervical spinal cord in the macaque monkey. J Comp Neurol 381:307-319. CrossRef Medline 
Galea MP, Darian-Smith I (1997b) Corticospinal projection patterns following unilateral section of the cervical spinal cord in the newborn and juvenile macaque monkey. J Comp Neurol 381:282-306. CrossRef Medline

García-Alías G, Barkhuysen S, Buckle M, Fawcett JW (2009) Chondroitinase $A B C$ treatment opens a window of opportunity for task-specific rehabilitation. Nat Neurosci 12:1145-1151. CrossRef Medline

Ghosh A, Haiss F, Sydekum E, Schneider R, Gullo M, Wyss MT, Mueggler T, Baltes C, Rudin M, Weber B, Schwab ME (2010) Rewiring of hindlimb corticospinal neurons after spinal cord injury. Nat Neurosci 13:97-104. CrossRef Medline

Grafen A, Hails R (2002) Modern statistics for the life sciences. Oxford: Oxford UP.

Hoenig JM, Heisey DM (2001) The abuse of power: the pervasive fallacy of power calculations for data analysis. Am Stat 55:19-24. CrossRef

Isa T, Kinoshita M, Nishimura Y (2013) Role of direct vs. indirect pathways from the motor cortex to spinal motoneurons in the control of hand dexterity. Front Neurol 4:191. CrossRef Medline

Iwamura Y (2000) Bilateral receptive field neurons and callosal connections in the somatosensory cortex. Philos Trans R Soc Lond Biol Sci 355:267273. CrossRef Medline

Karimi-Abdolrezaee S, Billakanti R (2012) Reactive astrogliosis after spinal cord injury-beneficial and detrimental effects. Mol Neurobiol 46:251264. CrossRef Medline

Killackey HP, Gould HJ 3rd, Cusick CG, Pons TP, Kaas JH (1983) The relation of corpus callosum connections to architectonic fields and body surface maps in sensorimotor cortex of New and Old World monkeys. J Comp Neurol 219:384-419. CrossRef Medline

Kuypers HG (1960) Central cortical projections to motor and somatosensory cell groups. An experimental study in the rhesus monkey. Brain 83:161-184. CrossRef Medline

Kuypers HG (1981) Anatomy of the descending pathways. In: Handbook of physiology - the nervous system II (Brookhart JM, Mountcastle VB, eds), pp 597-666. Bethesda, MD: American Physiological Society.

LaMotte CC, Kapadia SE, Shapiro CM (1991) Central projections of the sciatic, saphenous, median, and ulnar nerves of the rat demonstrated by transganglionic transport of choleragenoid-HRP (B-HRP) and wheat germ agglutinin-HRP (WGA-HRP). J Comp Neurol 311:546-562. CrossRef Medline

Lee T, Kim U (2012) Descending projections from the dysgranular zone of rat primary somatosensory cortex processing deep somatic input. J Comp Neurol 520:1021-1046. CrossRef Medline

Lemon RN (2008) Descending pathways in motor control. Annu Rev Neurosci 31:195-218. CrossRef Medline

Lemon RN, Griffiths J (2005) Comparing the function of the corticospinal system in different species: organizational differences for motor specialization? Muscle Nerve 32:261-279. CrossRef Medline

Levine M, Ensom MH (2001) Post hoc power analysis: An idea whose time has passed? Pharmacotherapy 21:405-409. CrossRef Medline
Liu K, Lu Y, Lee JK, Samara R, Willenberg R, Sears-Kraxberger I, Tedeschi A, Park KK, Jin D, Cai B, Xu B, Connolly L, Steward O, Zheng B, He Z (2010) PTEN deletion enhances the regenerative ability of adult corticospinal neurons. Nat Neurosci 13:1075-1081. CrossRef Medline

Miranda JD, White LA, Marcillo AE, Willson CA, Jagid J, Whittemore SR (1999) Induction of Eph B3 after spinal cord injury. Exp Neurol 156: 218-222. CrossRef Medline

Morecraft RJ, Ge J, Stilwell-Morecraft KS, McNeal DW, Pizzimenti MA, Darling WG (2013) Terminal distribution of the corticospinal projection from the hand/arm region of the primary motor cortex to the cervical enlargement in rhesus monkey. J Comp Neurol 521:4205-4235. CrossRef Medline

Murray EA, Coulter JD (1981) Organization of corticospinal neurons in the monkey. J Comp Neurol 195:339-365. CrossRef Medline

Newman JA, Bergelson J, Grafen A (1997) Blocking factors and hypothesis tests in ecology: is your statistics text wrong? Ecology 78:1312-1320. CrossRef

Nudo RJ, Masterton RB (1990) Descending pathways to the spinal cord, IV: some factors related to the amount of cortex devoted to the corticospinal tract. J Comp Neurol 296:584-597. CrossRef Medline

Ralston DD, Ralston HJ 3rd (1985) The terminations of corticospinal tract axons in the macaque monkey. J Comp Neurol 242:325-337. CrossRef Medline

Rhodes KE, Fawcett JW (2004) Chondroitin sulphate proteoglycans: preventing plasticity or protecting the CNS? J Anat 204:33-48. CrossRef Medline

Rolls A, Shechter R, Schwartz M (2009) The bright side of the glial scar in CNS repair. Nat Rev Neurosci 10:235-241. CrossRef Medline

Rosenzweig ES, Brock JH, Culbertson MD, Lu P, Moseanko R, Edgerton VR, Havton LA, Tuszynski MH (2009) Extensive spinal decussation and bilateral termination of cervical corticospinal projections in rhesus monkeys. J Comp Neurol 513:151-163. CrossRef Medline

Rosenzweig ES, Courtine G, Jindrich DL, Brock JH, Ferguson AR, Strand SC, Nout YS, Roy RR, Miller DM, Beattie MS, Havton LA, Bresnahan JC, Edgerton VR, Tuszynski MH (2010) Extensive spontaneous plasticity of corticospinal projections after primate spinal cord injury. Nat Neurosci 13:1505-1510. CrossRef Medline

Russell WM (1995) The development of the three Rs concept. Altern Lab Anim 23:298-304. Medline

Schieber MH (2007) Chapter 2. Comparative anatomy and physiology of the corticospinal system. Handb Clin Neurol 82:15-37. CrossRef Medline

Valtschanoff JG, Weinberg RJ, Rustioni A (1993) Amino acid immunoreactivity in corticospinal terminals. Exp Brain Res 93:95-103. Medline

Yang H, Lu P, McKay HM, Bernot T, Keirstead H, Steward O, Gage FH, Edgerton VR, Tuszynski MH (2006) Endogenous neurogenesis replaces oligodendrocytes and astrocytes after primate spinal cord injury. J Neurosci 26:2157-2166. CrossRef Medline 